\title{
STABILITY OF LIMIT CYCLE IN A DELAYED MODEL FOR TUMOR IMMUNE SYSTEM COMPETITION WITH NEGATIVE IMMUNE RESPONSE
}

\author{
RADOUANE YAFIA
}

Received 20 December 2005; Accepted 11 April 2006

This paper is devoted to the study of the stability of limit cycles of a system of nonlinear delay differential equations with a discrete delay. The system arises from a model of population dynamics describing the competition between tumor and immune system with negative immune response. We study the local asymptotic stability of the unique nontrivial equilibrium of the delay equation and we show that its stability can be lost through a Hopf bifurcation. We establish an explicit algorithm for determining the direction of the Hopf bifurcation and the stability or instability of the bifurcating branch of periodic solutions, using the methods presented by Diekmann et al.

Copyright (c) 2006 Radouane Yafia. This is an open access article distributed under the Creative Commons Attribution License, which permits unrestricted use, distribution, and reproduction in any medium, provided the original work is properly cited.

\section{Introduction}

We consider in this paper the model which provides a description of tumor cells in competition with the immune system. This description is described by many authors, using ordinary and delayed differential equations to model the competition between immune system and tumor, in particular see $[20,24,25]$. Other similar models provide a description of the modelling, analysis, and control of tumor immune system interaction, see, $[17,26,29]$.

Other authors use kinetic equations to model the competition between immune system and tumor. Although they give a complex description in comparison with other simpler models, they are, for example, needed to model the differences of virulence between viruses, see $[1-5,10]$. Several other fields of biology use kinetic equations, for instance $[12,13]$ give a kinetic approach to describe population dynamics, [2] deals with the development of suitable general mathematical structures including a large variety of Boltzmann-type models.

The reader interested in a more complete bibliography about the evolution of a cell, and the pertinent role that has cellular phenomena to direct the body towards the recovery

Hindawi Publishing Corporation

Discrete Dynamics in Nature and Society

Volume 2006, Article ID 58463, Pages 1-13

DOI 10.1155/DDNS/2006/58463 
2 Stability of limit cycle in tumor immune system

\begin{tabular}{|c|c|c|c|c|}
\hline & $k_{1}$ & & $k_{2}$ & \\
\hline$E+T$ & $\underset{k_{-1}}{\rightleftarrows}$ & $C$ & $k_{3}$ & $\begin{array}{l}E+T^{*} \\
E^{*}+T\end{array}$ \\
\hline
\end{tabular}

Figure 2.1. Kinetic scheme describing interactions between ECs and TCs (see [20]).

or towards the illness, is addressed to $[6,21]$. A detailed description of virus, antivirus, body dynamics can be found in the references $[8,14,27,28]$.

The mathematical model with which we are dealing was proposed in a recent paper by Gałach [20]. In this paper, the author developed a new simple model with one delay of tumor immune system competition, this idea is inspired from [25] and he recalled some numerical results in [25] in order to compare them with those obtained in his paper [20].

\section{Mathematical model}

The model proposed in [25] describes the response of effector cells (ECs) to the growth of tumor cells (TCs). This model differs from others because it takes into account the penetration of TCs by ECs, which simultaneously causes the inactivation of ECs. It is assumed that interactions between ECs and TCs in vitro can be described by the kinetic scheme shown in Figure 2.1, where $E, T, C, E^{*}$, and $T^{*}$ are the local concentrations of ECs, TCs, EC-TC complexes, inactivated ECs, and "lethally hit" TCs, respectively, $k_{1}$ and $k_{-1}$ denote the rates of bindings of ECs to TCs and the detachment of ECs from TCs without damaging them, $k_{2}$ is the rate at which EC-TC interactions program TCs for lysis, and $k_{3}$ is the rate at which EC-TC interactions inactivate ECs.

Kuznetsov and Taylor model is as follows:

$$
\begin{gathered}
\frac{d E}{d t}=s+F(C, T)-d_{1} E-k_{1} E T+\left(k_{-1}+k_{2}\right) C \\
\frac{d T}{d t}=a T(1-b T)-k_{1} E T+\left(k_{-1}+k_{3}\right) C \\
\frac{d C}{d t}=k_{1} E T-\left(k_{-1}+k_{2}+k_{3}\right) C \\
\frac{d E^{*}}{d t}=k_{3} C-d_{2} E^{*} \\
\frac{d T^{*}}{d t}=k_{2} C-d_{3} T^{*}
\end{gathered}
$$

where $s$ is the normal (i.e., not increased by the presence of the tumor) rate of the flow of adult ECs into the tumor site, $F(C, T)$ describes the accumulation of ECs in the tumor site, $d_{1}, d_{2}$, and $d_{3}$ are the coefficients of the processes of destruction and migration for $E, E^{*}$, and $T^{*}$, respectively, $a$ is the coefficient of the maximal growth of tumor, and $b$ is the environment capacity.

In [25], it is claimed that experimental observations motivate the approximation $d C / d t \approx 0$. Therefore, it is assumed that $C \approx K E T$, where $K=k_{1} /\left(k_{2}+k_{3}+k_{-1}\right)$, and the model can be reduced to two equations which describe the behavior of ECs and TCs only. 
Moreover, in [20], it is suggested that the function $F$ should be in the following form: $F(C, T)=F(E, T)=\theta E T$. Therefore, the model (2.1) takes the form

$$
\begin{gathered}
\frac{d E}{d t}=s+\alpha_{1} E T-d E, \\
\frac{d T}{d t}=a T(1-b T)-n E T,
\end{gathered}
$$

where $\alpha_{1}=\theta-m$, and $a, b, s$ have the same meaning as in $(2.1) ; n=K / k_{2}, m=K / k_{3}$, $d=d_{1}$. All coefficients except $\alpha_{1}$ are positive. The sign of $\alpha_{1}$ depends on the relation between $\theta$ and $m$. If the stimulation coefficient of the immune system exceeds the neutralization coefficient of ECs in the process of the formation of EC-TC complexes, then $\alpha_{1}>0$. We use the dimensionless form of model (2.2):

$$
\begin{gathered}
\frac{d x}{d t}=\sigma+\omega x y-\delta x, \\
\frac{d y}{d t}=\alpha y(1-\beta y)-x y,
\end{gathered}
$$

where $x$ denotes the dimensionless density of ECs, $y$ stands for dimensionless density of the population of TCs, $\alpha=a / K k_{2} T_{0}, \beta=b T_{0}, \delta=d / K k_{2} T_{0}, \sigma=s / n E_{0} T_{0}$, and $\omega=\alpha_{1} / n$ is the immune response to the appearance of the tumor cells, $E_{0}$ and $T_{0}$ are the initial conditions. In [20], the author studies the existence, uniqueness, and nonnegativity of solutions and he shows the nonexistence of nonnegative periodic solution of system (2.3).

For $\omega<0, \alpha \delta>\sigma$ and $\alpha^{2}(\beta \delta-\omega)^{2}+4 \alpha \beta \sigma \omega>0$, (2.3) has two possible nonnegative steady states $P_{0}$ and $P_{2}$, where the first is unstable and the second is stable (see [20]).

The delayed mathematical model corresponding to $(2.3)$ is given by the following system [20]:

$$
\begin{gathered}
\frac{d x}{d t}=\sigma+\omega x(t-\tau) y(t-\tau)-\delta x, \\
\frac{d y}{d t}=\alpha y(1-\beta y)-x y
\end{gathered}
$$

where the parameter $\tau$ is the time delay which the immune system needs to develop a suitable response after the recognition of nonself cells (see [20]). Time delays in connection with the tumor growth also appear in $[6,7,9,18,19]$.

The existence and uniqueness of solutions of system (2.4) for every $t>0$ are established in [20], and in the same paper it is shown that

(1) if $\omega \geq 0$, these solutions are nonnegative for any nonnegative initial conditions (biologically realistic case),

(2) if $\omega<0$, there exists nonnegative initial condition such that the solution becomes negative in a finite-time interval. 
Our goal in this paper is to consider the case (2) when the immune response is negative (i.e., $\omega<0)$ with the following conditions: $\alpha \delta>\sigma$ and $\alpha^{2}(\beta \delta-\omega)^{2}+4 \alpha \beta \sigma \omega>0$. We give some local stability results for the unique nontrivial equilibrium $P_{2}$ and that it undergoes a Hopf bifurcation (see [32]). We establish a systematic criteria for determining the direction of Hopf bifurcation and the stability or instability of the bifurcating branch of periodic solutions. The case (1) when the immune response is positive (i.e., $\omega>0$ ) is treated in $[30,31]$.

This paper is organized as follows. In Sections 3 and 4, we establish some results on the stability of the nontrivial steady states of the delayed system (2.4). The existence of a critical value of the delay in which the non-trivial steady state changes stability is investigated. Based on the Hopf bifurcation theorem, we show the occurrence of Hopf bifurcation as the delay crosses some critical value of the parameter delay (see [32]). The main result of this paper is given in Section 5, we establish a systematic criteria for determining the direction of Hopf bifurcation and the stability or instability of the bifurcating branch of periodic solutions.

\section{Steady states and stability for positive delays}

Consider the system (2.4), and suppose that $\omega<0, \alpha \delta>\sigma$, and $\alpha^{2}(\beta \delta-\omega)^{2}+4 \alpha \beta \sigma \omega>0$.

Then, system (2.4) has two equilibrium points $P_{0}=(\sigma / \delta, 0)$ and $P_{2}=\left(x_{2}, y_{2}\right)$, where

$$
x_{2}=\frac{-\alpha(\beta \delta-\omega)+\sqrt{\Delta}}{2 \omega}, \quad y_{2}=\frac{\alpha(\beta \delta+\omega)-\sqrt{\Delta}}{2 \alpha \beta \omega}
$$

with $\Delta=\alpha^{2}(\beta \delta-\omega)^{2}+4 \alpha \beta \sigma \omega$.

We will study the stability of the nontrivial equilibrium point $P_{2}$.

Let $u=x-x_{2}$ and $v=y-y_{2}$. By linearizing system (2.4) around the nontrivial equilibrium point $P_{2}$, we obtain the following linear system:

$$
\begin{gathered}
\frac{d u}{d t}=\omega x_{2} v(t-\tau)-\omega y_{2} u(t-\tau),-\delta u \\
\frac{d v}{d t}=-y_{2} u+\left(\alpha-2 \alpha \beta y_{2}-x_{2}\right) v
\end{gathered}
$$

The characteristic equation of (3.2) has the form

$$
W(\lambda, \tau)=\lambda^{2}+p \lambda+r+(s \lambda+q) e^{-\lambda \tau}=0,
$$

where $p=\delta+\alpha \beta y_{2}>0, r=\delta \alpha \beta y_{2}>0, s=-\omega y_{2}>0$, and $q=\alpha \omega y_{2}\left(1-2 \beta y_{2}\right)$.

The stability of the equilibrium point $P_{2}$ is a result of the localization of the roots of the equation

$$
W(\lambda, \tau)=0
$$

then we have the following theorem. 
Theorem 3.1. Assume $\alpha \delta>\sigma, \alpha>0$, and $\beta>0$ are close enough to 0 . Then, there exists $\tau_{l}>0$ such that $P_{2}$ is asymptotically stable for $\tau<\tau_{l}$ and unstable for $\tau>\tau_{l}$, where

$$
\begin{gathered}
\tau_{l}=\frac{1}{\zeta_{l}} \arccos \left\{\frac{q\left(\zeta_{l}^{2}-r\right)-p s \zeta_{l}^{2}}{s^{2} \zeta_{l}^{2}+q^{2}}\right\} \\
\zeta_{l}^{2}=\frac{1}{2}\left(s^{2}-p^{2}+2 r\right)+\frac{1}{2}\left[\left(s^{2}-p^{2}+2 r\right)^{2}-4\left(r^{2}-q^{2}\right)\right]^{1 / 2} .
\end{gathered}
$$

For the proof of Theorem 3.1, we need the following lemma.

Lemma 3.2 [11]. Consider the equation

$$
\lambda^{2}+p \lambda+r+(s \lambda+q) e^{-\lambda \tau}=0
$$

where $p, r, q$, and s are real numbers.

Let the following hypotheses hold:

$\left(\mathrm{H}_{1}\right) p+s>0$,

$\left(\mathrm{H}_{2}\right) q+r>0$,

$\left(\mathrm{H}_{3}\right) r^{2}-q^{2}<0$ or $\left(s^{2}-p^{2}+2 r>0\right.$, and $\left.\left(s^{2}-p^{2}+2 r\right)^{2}=4\left(r^{2}-q^{2}\right)\right)$.

If $\left(\mathrm{H}_{1}\right)-\left(\mathrm{H}_{3}\right)$ hold, then when $\tau \in\left[0, \tau_{l}\right)$, all roots of (3.6) have negative real parts, when $\tau=\tau_{l}$, (3.6) has a pair of purely imaginary roots $\pm i \zeta_{l}$, and when $\tau>\tau_{l},(3.6)$ has at least one root with positive real part, where $\tau_{l}$ and $\zeta_{l}$ are defined in Theorem 3.1.

Proof of Theorem 3.1. From the expressions of $p, q$, s, and $r$, we have $p+s>0$ and

$$
q+r=-\alpha(\omega+\delta \beta) y_{2}+2(\alpha \delta-\sigma)
$$

As $\beta$ is close enough to 0 , we have $\omega / \beta<-\delta$.

From the hypothesis $\alpha \delta>\sigma$, we deduce that $q+r>0$.

Therefore, the hypotheses $\left(\mathrm{H}_{1}\right),\left(\mathrm{H}_{2}\right)$ of Lemma 3.2 are satisfied. Then all roots of the characteristic (3.3) have negative real parts for $\tau=0$ and the steady state $P_{2}$ is asymptotically stable for $\tau=0$. By Rouche's theorem, it follows that the roots of (3.3) have negative real parts for some critical value of the delay $\tau$.

We want to determine if the real part of some root increases to reach zero and eventually becomes positive as $\tau$ varies. If $i \zeta$ is a root of (3.3), then

$$
-\zeta^{2}+i p \zeta+i s \zeta(\cos (\tau \zeta)+i \sin (\tau \zeta))+r+q(\cos (\tau \zeta)+i \sin (\tau \zeta))=0
$$

Separating the real and imaginary parts, we have

$$
\begin{gathered}
-\zeta^{2}+r=-q \cos (\tau \zeta)+s \zeta \sin (\tau \zeta), \\
p \zeta=-s \zeta \cos (\tau \zeta)-q \sin (\tau \zeta) .
\end{gathered}
$$

It follows that $\zeta$ satisfies

$$
\zeta^{4}-\left(s^{2}-p^{2}+2 r\right) \zeta^{2}+\left(r^{2}-q^{2}\right)=0 .
$$


6 Stability of limit cycle in tumor immune system

The two roots of the above equation can be expressed as follows:

$$
\zeta^{2}=\frac{1}{2}\left(s^{2}-p^{2}+2 r\right) \pm \frac{1}{2}\left[\left(s^{2}-p^{2}+2 r\right)^{2}-4\left(r^{2}-q^{2}\right)\right]^{1 / 2} .
$$

As $r^{2}-q^{2}=\alpha^{2} y_{2}^{2}\left(\delta^{2} \beta^{2}-\omega^{2}\left(1-2 \beta y_{2}\right)^{2}\right)$, the sign of $r^{2}-q^{2}$ is deduced from the sign of $\left(\delta \beta-\omega^{2}\left(1-2 \beta y_{2}\right)\right)=(2 \alpha \beta \delta-\sqrt{\Delta}) / \alpha$ which is negative (because $\beta$ is very small and $\alpha>0)$.

Therefore, $r^{2}-q^{2}<0$ and the hypothesis $\left(\mathrm{H}_{3}\right)$ of Lemma 3.2 is satisfied.

From Lemma 3.2, the unique solution of (3.10) has the following form:

$$
\zeta_{l}^{2}=\frac{1}{2}\left(s^{2}-p^{2}+2 r\right)+\frac{1}{2}\left[\left(s^{2}-p^{2}+2 r\right)^{2}-4\left(r^{2}-q^{2}\right)\right]^{1 / 2}
$$

and there exists a unique critical value

$$
\tau_{l}=\frac{1}{\zeta_{l}} \arccos \left\{\frac{q\left(\zeta_{l}^{2}-r\right)-p s \zeta_{l}^{2}}{s^{2} \zeta_{l}^{2}+q^{2}}\right\}
$$

such that the equilibrium point $P_{2}$ is asymptotically stable for $\tau \in\left[0, \tau_{l}\right)$ and unstable for $\tau>\tau_{l}$. For $\tau=\tau_{l}$, the characteristic (3.3) has a pair of purely imaginary roots $\pm i \zeta_{l}$.

In Section 4, we will study the occurrence of Hopf bifurcation when the delay passes through the critical value of the delay $\tau=\tau_{l}$.

Let $z(t)=(u(t), v(t))=(x(t), y(t))-\left(x_{2}, y_{2}\right)$, then the system (2.4) is written as a functional differential equation $(\mathrm{FDE})$ in $C:=C\left([-\tau, 0], \mathbb{R}^{2}\right)$ :

$$
\frac{d z(t)}{d t}=L(\tau) z_{t}+f\left(z_{t}, \tau\right)
$$

where $L(\tau): C \rightarrow \mathbb{R}^{2}$ is a linear operator and $f: C \times \mathbb{R} \rightarrow \mathbb{R}^{2}$ are given, respectively, by

$$
\begin{gathered}
L(\tau) \varphi=\left(\begin{array}{c}
\omega y_{2} \varphi_{1}(-\tau)+\omega x_{2} \varphi_{2}(-\tau)-\delta \varphi_{1}(0) \\
-y_{2} \varphi_{1}(0)+\left(\alpha-2 \alpha \beta y_{2}-x_{2}\right) \varphi_{2}(0)
\end{array}\right), \\
f(\varphi, \tau)=\left(\begin{array}{c}
\sigma+\omega \varphi_{1}(-\tau) \varphi_{2}(-\tau)+\omega x_{2} y_{2}-\delta x_{2} \\
-\alpha \beta \varphi_{2}^{2}(0)+\alpha y_{2}-\alpha \beta y_{2}^{2}-\varphi_{1}(0) \varphi_{2}(0)-x_{2} y_{2}
\end{array}\right)
\end{gathered}
$$

for $\varphi=\left(\varphi_{1}, \varphi_{2}\right) \in C$.

\section{Hopf bifurcation occurrence}

According to the Hopf bifurcation theorem [22], we come to the main result of this paper.

Theorem 4.1. Assume $\alpha \delta>\sigma, \alpha>0, \beta>0$, and $\beta$ are close enough to 0 . There exists $\varepsilon_{1}>0$ such that for each $0 \leq \varepsilon<\varepsilon_{1}$, (3.14) has a family of periodic solutions $p_{l}(\varepsilon)$ with period $T_{l}=$ $T_{l}(\varepsilon)$, for the parameter values $\tau=\tau(\varepsilon)$ such that $p_{l}(0)=P_{2}, T_{l}(0)=2 \pi / \zeta_{l}$, and $\tau(0)=\tau_{l}$, where $\tau_{l}$ and $\zeta_{l}$ are given, respectively, in (3.5). 
Proof. We apply the Hopf bifurcation theorem introduced in [22]. From the expression of $f$ in (3.14), we have

$$
f(0, \tau)=0, \quad \frac{\partial f(0, \tau)}{\partial \varphi}=0, \quad \forall \tau>0 .
$$

From (3.3) and Theorem 3.1, the characteristic equation (3.3) has a pair of simple imaginary roots $\lambda_{l}=i \zeta_{l}$ and $\bar{\lambda}_{l}=-i \zeta_{l}$ at $\tau=\tau_{l}$.

From (3.3), $W\left(\lambda_{l}, \tau_{l}\right)=0$ and $(\partial / \partial \lambda) W\left(\lambda_{l}, \tau_{l}\right)=2 i \zeta_{l}+p+\left(s-\tau\left(i s \zeta_{l}+q\right)\right) e^{-i \zeta_{l} \tau_{l}} \neq 0$. According to the implicit function theorem, there exists a complex function $\lambda=\lambda(\tau)$ defined in a neighborhood of $\tau_{l}$, such that $\lambda\left(\tau_{l}\right)=\lambda_{l}$ and $W(\lambda(\tau), \tau)=0$, and

$$
\begin{gathered}
\lambda^{\prime}(\tau)=-\frac{\partial W(\lambda, \tau) / \partial \tau}{\partial W(\lambda, \tau) / \partial \lambda} \quad \text { for } \tau \text { in a neighborhood of } \tau_{l}, \\
\lambda^{\prime}(\tau)=\frac{\lambda(s \lambda+q) e^{-\lambda \tau}}{2 \lambda+p+(s-\tau s \lambda-\tau q) e^{-\lambda \tau}} .
\end{gathered}
$$

From (3.3), (3.9), and (4.3), we obtain the following expression of $\lambda^{\prime}(\tau)$ for $\tau$ in a neighborhood of $\tau_{l}$ :

$$
\lambda^{\prime}(\tau)=-\lambda \frac{s \lambda^{3}+\left(s^{2} p+q\right) \lambda^{2}+(s r+p q) \lambda+q r}{\tau s \lambda^{3}+(s+\tau(s p+q)) \lambda^{2}+(2 q+\tau(s r+p q)) \lambda+p q-s r+q r} .
$$

Let $\lambda(\tau)=\kappa(\tau)+i \zeta(\tau)$ (where $\kappa$ and $\zeta$ are the real and imaginary parts of $\lambda$, resp.). From (4.4), we have

$\kappa^{\prime}(\tau)_{/ \tau=\tau_{l}}=\zeta_{l}^{2} \frac{s^{2} \zeta_{l}^{4}+\left(s q r(\tau-1)+2 q^{2}\right) \zeta_{l}^{2}+s r^{2}(q-s r)+p q^{2}(p+r)-q r(2 q+\tau(s r+p q))}{A^{2}+B^{2}}$,

where

$$
\begin{gathered}
A=-(s+\tau(s p+q)) \zeta_{l}^{2}+p q-s r+q r \\
B=-\tau s \zeta_{l}^{2}+(2 q+\tau(s r+p q)) \zeta_{l} .
\end{gathered}
$$

From the expression of $r$ and as $\beta$ is close to 0 , then $r$ is very small. From (4.5), we conclude that

$$
\kappa^{\prime}(\tau)_{/ \tau=\tau_{l}}>0 .
$$

Then, the transversality condition is verified, which completes the proof of Theorem 4.1.

\section{Direction of Hopf bifurcation}

For determining the direction of Hopf bifurcation, there exist many formulas, we cite

(1) the formulas using the theory of normal forms, see Hassard et al. [23], (2) Faria and Magalhães [16], (3) Diekmann [15]. In this section, we follow methods presented in [15], 
8 Stability of limit cycle in tumor immune system

where the direction and stability of the bifurcating branch are obtained by the Taylor expansion of the delay function $\tau$ that describes the parameter of bifurcation near the critical value $\tau_{l}$ (see Sections 3 and 4). Namely, this direction and stability are determined by the sign of the first nonzero term of Taylor expansion, that is,

$$
\tau(\varepsilon)=\tau_{l}+\tau_{2} \varepsilon^{2}+o\left(\varepsilon^{2}\right)
$$

and the sign of $\tau_{2}$ determines either the bifurcation is supercritical (if $\tau_{2}>0$ ) and periodic orbits exist for $\tau>\tau_{l}$, or it is subcritical (if $\tau_{2}<0$ ) and periodic orbits exist for $\tau<\tau_{l}$. The term $\tau_{2}$ may be calculated, see [15], using the formula

$$
\tau_{2}=\frac{\operatorname{Re}(c)}{\operatorname{Re}\left(q D_{2} M\left(i \zeta_{l}, \tau_{l}\right) p\right)},
$$

where $M$ is the characteristic matrix of (3.14) given by

$$
M(\lambda, \tau)=\left(\begin{array}{cc}
\lambda+\omega y_{2} e^{-\lambda \tau}+\delta & -\omega x_{2} e^{-\lambda \tau} \\
y_{2} & \lambda-\alpha+2 \alpha \beta y_{2}+x_{2}
\end{array}\right),
$$

$D_{2} M\left(i \zeta_{l}, \tau_{l}\right)$ denotes the derivative of $M$ with respect to $\tau$ at the critical point $\left(i \zeta_{l}, \tau_{l}\right)$, the constant $c$ is defined as follows:

$$
\begin{aligned}
c= & \frac{1}{2} q D_{1}^{3} f\left(0, \tau_{l}\right)\left(P^{2}(\theta), \bar{P}(\theta)\right) \\
& +q D_{1}^{2} f\left(0, \tau_{l}\right)\left(e^{0 .} M^{-1}\left(0, \tau_{l}\right) D_{1}^{2} f\left(0, \tau_{l}\right)(P(\theta), \bar{P}(\theta)), P(\theta)\right) \\
& +\frac{1}{2} q D_{1}^{2} f\left(0, \tau_{l}\right)\left(e^{2 i \zeta_{l} \cdot} M^{-1}\left(2 i \zeta_{l}, \tau_{l}\right) D_{1}^{2} f\left(0, \tau_{l}\right)(P(\theta), P(\theta)), \bar{P}(\theta)\right),
\end{aligned}
$$

where $f$ is the nonlinear part of (3.14), $D_{1}^{i} f, i=2,3$, denotes the ith derivative of $f$ with respect to $\varphi, P(\theta)$ denotes the eigenvector of $A, \bar{P}(\theta)$ denotes the conjugate eigenvector, and $p$ and $q$ are defined later.

Now, we describe all the above operators and vectors precisely. Let

$$
L:=L\left(\tau_{l}\right): C\left(\left[-\tau_{l}, 0\right], \mathbb{R}^{2}\right) \longrightarrow \mathbb{R}^{2}
$$

denote the linear part of (3.14). Using the Riesz representation theorem, one obtains, see [22],

$$
L \varphi=\int_{-\tau_{l}}^{0} d \eta(\theta) \varphi(\theta)
$$

where,

$$
d \eta(\theta)=\left(\begin{array}{cc}
-\omega y_{2} \delta\left(\theta+\tau_{l}\right)-\delta \delta(\theta) & \omega x_{2} \delta\left(\theta+\tau_{l}\right) \\
-y_{2} \delta(\theta) & \left(\alpha-2 \alpha \beta y_{2}-x_{2}\right) \delta(\theta)
\end{array}\right),
$$

$\delta(\cdot)$ denotes the Dirac function. 
Let $A$ denote the generator of semigroup generated by the linear part of (3.14). Then,

$$
A \varphi(\theta)= \begin{cases}\frac{d \varphi}{d \theta}(\theta) & \text { for } \theta \in\left[-\tau_{l}, 0\right) \\ L \varphi & \text { for } \theta=0\end{cases}
$$

where $\varphi \in C\left(\left[-\tau_{l}, 0\right], \mathbb{R}^{2}\right)$.

To study the direction of Hopf bifurcation, one needs to calculate the second and third derivatives of nonlinear part of (3.14):

$$
\begin{gathered}
D_{1}^{2} f\left(0, \tau_{l}\right) \psi \chi=\left(\begin{array}{c}
\omega \psi_{1}\left(-\tau_{l}\right) \chi_{2}\left(-\tau_{l}\right)+\omega \psi_{2}\left(-\tau_{l}\right) \chi_{1}\left(-\tau_{l}\right) \\
-\psi_{1}(0) \chi_{2}(0)-\psi_{2}(0) \chi_{1}(0)-2 \alpha \beta \psi_{2}(0) \chi_{2}(0)
\end{array}\right), \\
D_{1}^{3} f\left(0, \tau_{l}\right)=0,
\end{gathered}
$$

$\psi=\left(\psi_{1}, \psi_{2}\right), \chi=\left(\chi_{1}, \chi_{2}\right) \in C\left(\left[-\tau_{l}, 0\right], \mathbb{R}^{2}\right)$.

As $\left(i \zeta_{l}, \tau_{l}\right)$ is a solution of $(3.3)$, then $i \zeta_{l}$ is an eigenvalue of $A$ and there is an eigenvector of the form $P(\theta)=p e^{i \zeta_{l} \theta}$ and $p_{i}, i=1,2$ are complex numbers which satisfy the following system of equations:

$$
M\left(i \zeta_{l}, \tau_{l}\right) p=0
$$

Then one may assume that

$$
p_{2}=1
$$

and calculate

$$
p_{1}=\frac{\alpha-2 \alpha \beta y_{2}-x_{2}-i \zeta_{l}}{y_{2}}
$$

Now, consider $A^{*}$, that is, an operator conjugated to $A, A^{*}: C\left([0,1], \mathbb{R}^{2}\right) \rightarrow \mathbb{R}^{2}$, defined by

$$
A^{*} \psi(s)= \begin{cases}-\frac{d \psi}{d s}(s) & \text { for } s \in(0,1] \\ -\int_{-\tau_{l}}^{0} \psi(-s) d \eta(s) & \text { for } s=0,\end{cases}
$$

$\psi=\left(\psi_{1}, \psi_{2}\right) \in C\left(\left[0, \tau_{l}\right], \mathbb{R}^{2}\right)$.

Let $Q(s)=q e^{i \zeta_{l} s}$ be the eigenvector for $A^{*}$ associated to eigenvalue $i \zeta_{l}, q=\left(q_{1}, q_{2}\right)^{T}$. One needs to choose $q$ such that the inner product (see [22])

$$
\langle Q, \bar{P}\rangle=Q(0) \bar{P}(0)-\int_{-1}^{0} \int_{0}^{\theta} Q(\xi-\theta) d \eta(\theta) \bar{P}(\xi) d \xi
$$


10 Stability of limit cycle in tumor immune system

is equal to 1 . Therefore,

$$
q_{2}=0
$$

leads to

$$
q_{1}=\frac{e^{-\zeta_{l} \tau_{l}}}{X-i Y}
$$

where

$$
\begin{gathered}
X=\frac{\alpha-2 \alpha \beta y_{2}-x_{2}}{y_{2}} \cos \left(\zeta_{l} \tau_{l}\right)+\frac{\zeta_{l}}{y_{2}} \sin \left(\zeta_{l} \tau_{l}\right)+\tau_{l} \omega\left(-\alpha+2 \alpha \beta y_{2}+2 x_{2}\right), \\
Y=-\frac{\alpha-2 \alpha \beta y_{2}-x_{2}}{y_{2}} \sin \left(\zeta_{l} \tau_{l}\right)-\frac{\zeta_{l}}{y_{2}} \cos \left(\zeta_{l} \tau_{l}\right)+\zeta_{l} \tau_{l} \omega .
\end{gathered}
$$

From (5.9), we have

$$
\begin{gathered}
D_{1}^{2} f\left(0, \tau_{l}\right)(P(\theta), \bar{P}(\theta))=\left(\begin{array}{c}
2 \omega \operatorname{Re}\left(p_{1}\right) \\
-2 \operatorname{Re}\left(p_{1}\right)-2 \alpha \beta
\end{array}\right), \\
D_{1}^{2} f_{0}\left(0, \tau_{0}\right)(P(\theta), P(\theta))\left(\begin{array}{c}
2 \omega p_{1} e^{-2 i \zeta_{l} \tau_{l}} \\
-2 p_{1}-2 \alpha \beta
\end{array}\right), \\
\frac{1}{2} q D_{1}^{3} f\left(0, \tau_{l}\right)\left(P^{2}(\theta), \bar{P}(\theta)\right)=0 .
\end{gathered}
$$

From the expression of $M$, we have

$$
\begin{gathered}
M^{-1}\left(0, \tau_{l}\right)=W^{-1}\left(0, \tau_{l}\right)\left(\begin{array}{cc}
-\alpha+2 \alpha \beta y_{2}+x_{2} & \omega x_{2} \\
-y_{2} & \omega y_{2}+\delta
\end{array}\right), \\
M^{-1}\left(2 i \zeta_{l}, \tau_{l}\right)=W^{-1}\left(2 i \zeta_{l}, \tau_{l}\right)\left(\begin{array}{cc}
2 i \zeta_{l}-\alpha+2 \alpha \beta y_{2}+x_{2} & \omega x_{2} e^{-2 i \zeta_{l} \tau_{l}} \\
-y_{2} & 2 i \zeta_{0}+\omega y_{2} e^{-2 i \zeta_{l} \tau_{l}}+\delta
\end{array}\right) .
\end{gathered}
$$

From (5.19) and (5.21), we have

$$
\begin{aligned}
q D_{1}^{2} f & \left(0, \tau_{l}\right)\left(e^{0} \cdot M^{-1}\left(0, \tau_{l}\right) D_{1}^{2} f\left(0, \tau_{l}\right)(P(\theta), \bar{P}(\theta)), P(\theta)\right) \\
& =W\left(0, \tau_{l}\right)^{-1} \frac{\omega}{X^{2}+Y^{2}}[X+i Y][B+i C],
\end{aligned}
$$

where

$$
\begin{aligned}
B= & 2 \omega\left(-\alpha+2 \alpha \beta y_{2}\right) \operatorname{Re}\left(p_{1}\right) \\
& -2 \omega \alpha \beta x_{2}-\left(\omega y_{2}+\delta\right)\left(2 \operatorname{Re}\left(p_{1}\right)+2 \alpha \beta\right) \operatorname{Re}\left(p_{1}\right)-2 \omega y_{2} \operatorname{Re}\left(p_{1}\right)^{2}, \\
C= & -\left(\omega y_{2}+\delta\right)\left(2 \operatorname{Re}\left(p_{1}\right)+2 \alpha \beta\right) \operatorname{Im}\left(p_{1}\right)-2 \omega y_{2} \operatorname{Re}\left(p_{1}\right) \operatorname{Im}\left(p_{1}\right) .
\end{aligned}
$$


Then

$$
\begin{aligned}
& \operatorname{Re}\left(q D_{1}^{2} f\left(0, \tau_{l}\right)\left(e^{0 \cdot} M^{-1}\left(0, \tau_{l}\right) D_{1}^{2} f\left(0, \tau_{l}\right)(P(\theta), \bar{P}(\theta)), P(\theta)\right)\right) \\
& \quad=W\left(0, \tau_{l}\right)^{-1} \frac{\omega}{X^{2}+Y^{2}}[X B-Y C] . \\
& \frac{1}{2} q D_{1}^{2} f\left(0, \tau_{l}\right)\left(e^{2 i \zeta_{l} \cdot} M^{-1}\left(2 i \zeta_{l}, \tau_{l}\right) D_{1}^{2} f\left(0, \tau_{l}\right)(P(\theta), P(\theta)), \bar{P}(\theta)\right) \\
& \quad=\frac{\omega}{2} \frac{(H E-K F)+i(H F+K E)}{\left(X^{2}+Y^{2}\right)\left|W\left(2 i \zeta_{l}, \tau_{l}\right)\right|^{2}} .
\end{aligned}
$$

Then

$$
\begin{aligned}
\operatorname{Re}( & \left.\frac{1}{2} q D_{1}^{2} f\left(0, \tau_{l}\right)\left(e^{2 i \zeta_{l} \cdot} M^{-1}\left(2 i \zeta_{l}, \tau_{l}\right) D_{1}^{2} f\left(0, \tau_{l}\right)(P(\theta), P(\theta)), \bar{P}(\theta)\right)\right) \\
& =\frac{\omega}{2} \frac{H E-K F}{\left(X^{2}+Y^{2}\right)\left|W\left(2 i \zeta_{l}, \tau_{l}\right)\right|^{2}}
\end{aligned}
$$

where

$$
\begin{aligned}
H= & X\left(-4 \zeta_{l}^{2}+r+q \cos \left(2 \zeta_{l} \tau_{l}\right)+2 s \zeta_{l} \sin \left(2 \zeta_{l} \tau_{l}\right)\right)-Y\left(2 p \zeta_{l}+2 s \zeta_{l} \cos \left(2 \zeta_{l} \tau_{l}\right)-q \sin \left(2 \zeta_{l} \tau_{l}\right)\right), \\
K= & Y\left(-4 \zeta_{l}^{2}+r+q \cos \left(2 \zeta_{l} \tau_{l}\right)+2 s \zeta_{l} \sin \left(2 \zeta_{l} \tau_{l}\right)\right)+X\left(2 p \zeta_{l}+2 s \zeta_{l} \cos \left(2 \zeta_{l} \tau_{l}\right)-q \sin \left(2 \zeta_{l} \tau_{l}\right)\right), \\
E= & \frac{2 \omega}{y_{2}}\left(\left(\alpha-2 \alpha \beta y_{2}-x_{2}\right)\left(-\alpha+2 \alpha \beta y_{2}\right)+2 \zeta_{l}^{2}\right) \\
& -\left(2\left|p_{1}\right|^{2}+\frac{2 \alpha \beta}{y_{2}}\left(\alpha-2 \alpha \beta y_{2}-x_{2}\right)\right)\left(\omega y_{2} \cos \left(2 \zeta_{l} \tau_{l}\right)+\delta\right) \\
& -\frac{\zeta_{l}}{y_{2}}\left(2 \zeta_{l}-\omega y_{2} \sin \left(2 \zeta_{l} \tau_{l}\right)\right)-2 \omega y_{2}\left|p_{1}\right|^{2} \cos \left(2 \zeta_{l} \tau_{l}\right)-2 \alpha \beta \omega x_{2}, \\
F= & \frac{2 \omega \zeta_{l}}{y_{2}}\left(3 \alpha-6 \alpha \beta y_{2}-2 x_{2}\right)-\left(2\left|p_{1}\right|^{2}+\frac{2 \alpha \beta}{y_{2}}\left(\alpha-2 \alpha \beta y_{2}-x_{2}\right)\right)\left(2 \zeta_{l}-\omega y_{2} \sin \left(2 \zeta_{l} \tau_{l}\right)\right) \\
& +\frac{\zeta_{l}}{y_{2}}\left(\omega y_{2} \cos \left(2 \zeta_{l} \tau_{l}\right)+\delta\right)+2 \omega y_{2}\left|p_{1}\right|^{2} \sin \left(2 \zeta_{l} \tau_{l}\right) .
\end{aligned}
$$

From the expressions of $M, P$, and $Q$, we have

$$
q D_{2} M\left(i \zeta_{l}, \tau_{l}\right) p=i \omega \zeta_{l} \frac{X\left(-\alpha+2 \alpha \beta y_{2}+2 x_{1}\right)-\zeta_{l} Y+i\left(Y\left(-\alpha+2 \alpha \beta y_{2}+2 x_{1}\right)+\zeta_{l} X\right)}{X^{2}+Y^{2}} .
$$

Then

$$
\operatorname{Re}\left(q D_{2} M\left(i \zeta_{l}, \tau_{l}\right) p\right)=-\omega \zeta_{l} \frac{Y\left(-\alpha+2 \alpha \beta y_{2}+2 x_{1}\right)+\zeta_{l} X}{X^{2}+Y^{2}}
$$


Then From (5.20), (5.25), (5.27), and (5.30), the expression of $\tau_{2}$ is given by

$$
\tau_{2}=-\frac{(1 / 2)(H E-K F) /\left|W\left(2 i \zeta_{l}, \tau_{l}\right)\right|^{2}+W\left(0, \tau_{l}\right)^{-1}[X B-Y C]}{\zeta_{l}\left[Y\left(-\alpha+2 \alpha \beta y_{2}+2 x_{1}\right)-\zeta_{l} X\right]}
$$

and we deduce the following result.

Theorem 5.1. Let $\tau_{2}$ be given in (5.31). Then,

(a) the Hopf bifurcation occurs as $\tau$ crosses $\tau_{l}$ to the right (supercritical Hopf bifurcation) if $\tau_{2}>0$ and to the left (subcritical Hopf bifurcation) if $\tau_{2}<0 ; 0$

(b) the bifurcating periodic solutions are stable if $\tau_{2}>0$ and unstable if $\tau_{2}<0$.

Note that Theorem 5.1 provides an explicit algorithm for detecting the direction and stability of Hopf bifurcation.

\section{References}

[1] J. Adam and N. Bellomo, A Survey of Models on Tumor Immune Systems Dynamics, Birkhäuser, Massachusetts, 1996.

[2] L. Arlotti, N. Bellomo, and E. De Angelis, Generalized kinetic (Boltzmann) models: mathematical structures and applications, Mathematical Models \& Methods in Applied Sciences 12 (2002), no. $4,567-591$.

[3] N. Bellomo and L. Preziosi, Modelling and mathematical problems related to tumor evolution and its interaction with the immune system, Mathematical and Computer Modelling 32 (2000), no. 3 4, 413-452.

[4] N. Bellomo and M. Pulvirenti (eds.), Modeling in Applied Sciences. A Kinetic Theory Approach, Modeling and Simulation in Science, Engineering and Technology, Birkhäuser Boston, Massachusetts, 2000.

[5] N. Bellomo and M. Pulvirenti (eds.), Special issue on the modeling in applied sciences by methods of transport and kinetic theory, Mathematical and Computer Modelling 12 (2002), 909-990.

[6] M. Bodnar and U. Foryś, Behaviour of solutions to Marchuk's model depending on a time delay, International Journal of Applied Mathematics and Computer Science 10 (2000), no. 1, 97-112.

[7] _ Periodic dynamics in a model of immune system, Applicationes Mathematicae 27 (2000), no. 1, 113-126.

[8] R. Bürger, The Mathematical Theory of Selection, Recombination, and Mutation, Wiley Series in Mathematical and Computational Biology, John Wiley \& Sons, Chichester, 2000.

[9] H. M. Byrne, The effect of time delays on the dynamics of avascular tumor growth, Mathematical Biosciences 144 (1997), no. 2, 83-117.

[10] M. A. J. Chaplain (ed.), Special issue on mathematical models for the growth, development and treatment of tumors, Mathematical Models and Methods in Applied Sciences 9 (1999).

[11] K. L. Cooke and Z. Grossman, Discrete delay, distributed delay and stability switches, Journal of Mathematical Analysis and Applications 86 (1982), no. 2, 592-627.

[12] L. Desvillettes and C. Prevots, Modelling in population dynamics through kinetic-like equations, preprint n. 99/19 of the University of Orléans, Département de mathématiques.

[13] L. Desvillettes, C. Prevots, and R. Ferrieres, Infinite dimensional reaction-diffusion for population dynamics, 2003.

[14] O. Diekmann and J. A. P. Heesterbeek, Mathematical Epidemiology of Infectious Diseases, Wiley Series in Mathematical and Computational Biology, John Wiley \& Sons, Chichester, 2000.

[15] O. Diekmann, S. A. van Gils, S. M. Verduyn Lunel, and H.-O. Walther, Delay Equations, Applied Mathematical Sciences, vol. 110, Springer, New York, 1995. 
[16] T. Faria and L. T. Magalhães, Normal forms for retarded functional-differential equations with parameters and applications to Hopf bifurcation, Journal of Differential Equations 122 (1995), no. $2,181-200$.

[17] U. Foryś, Marchuk's model of immune system dynamics with application to tumor growth, Journal of Theoretical Medicine 4 (2002), no. 1, 85-93.

[18] U. Foryś and M. Kolev, Time delays in proliferation and apoptosis for solid avascular tumor, preprint Institute of Applied Mathematics and Machanics, no. RW 02-10 (110), Warsaw University (2002).

[19] U. Foryś and A. Marciniak-Czochra, Delay logistic equation with diffusion, Proceedings of the 8th National Conference on Application of Mathematics in Biology and Medicine, Łajs, 2002, pp. 37-42.

[20] M. Gałach, Dynamics of the tumor-immune system competition — the effect of time delay, International Journal of Applied Mathematics and Computer Science 13 (2003), no. 3, 395-406.

[21] L. Greller, F. Tobin, and G. Poste, Tumor hetereogenity and progression: conceptual foundation for modeling, Invasion and Metastasis 16 (1996), 177-208.

[22] J. K. Hale and S. M. Verduyn Lunel, Introduction to Functional-Differential Equations, Applied Mathematical Sciences, vol. 99, Springer, New York, 1993.

[23] B. D. Hassard, N. D. Kazarinoff, and Y. H. Wan, Theory and Applications of Hopf Bifurcation, London Mathematical Society Lecture Note Series, vol. 41, Cambridge University Press, Cambridge, 1981.

[24] D. Kirschner and J. C. Panetta, Modeling immunotherapy of the tumor-immune interaction, Journal of Mathematical Biology 37 (1998), no. 3, 235-252.

[25] V. A. Kuznetsov, I. A. Makalkin, M. A. Taylor, and A. S. Perelson, Nonlinear dynamics of immunogenic tumors: parameter estimation and global bifurcation analysis, Bulletin of Mathematical Biology 56 (1994), no. 2, 295-321.

[26] H. Mayer, K. S. Zänker, and U. der Heiden, A basic mathematical model of the immune response, Chaos 5 (1995), no. 1, 155-161.

[27] M. A. Nowak and R. M. May, Virus Dynamics. Mathematical Principles of Immunology and Virology, Oxford University Press, Oxford, 2000.

[28] A. S. Perelson and G. Weisbuch, Immunology for physicists, Reviews of Modern Physics 69 (1997), no. $4,1219-1267$.

[29] J. Waniewski and P. Zhivkov, A simple mathematical model for tumor-immune system interactions, Proceedings of the 8th National Conference on Application of Mathematics in Biology and Medicine, Łajs, 2002, pp. 149-154.

[30] R. Yafia, Dynamics, Hopf bifurcation and stability analysis in a model for tumor-immune system competition with one delay, in preparation.

[31] _ Hopf bifurcation in a delayed model for tumor-immune system competition, in preparation.

[32] __ Hopf bifurcation in a delayed model for tumor-immune system competition with negative immune response, to appear in Discrete Dynamics in Nature and Society.

Radouane Yafia: Département de Mathématiques et Informatique, Faculté des Sciences,

Université Chouaib Doukkali, BP 20, El Jadida, Morocco

E-mail addresses: yafia@math.net; yafia_radouane@hotmail.com 


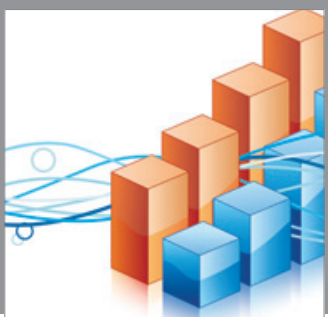

Advances in

Operations Research

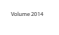

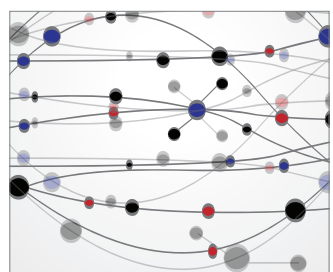

\section{The Scientific} World Journal
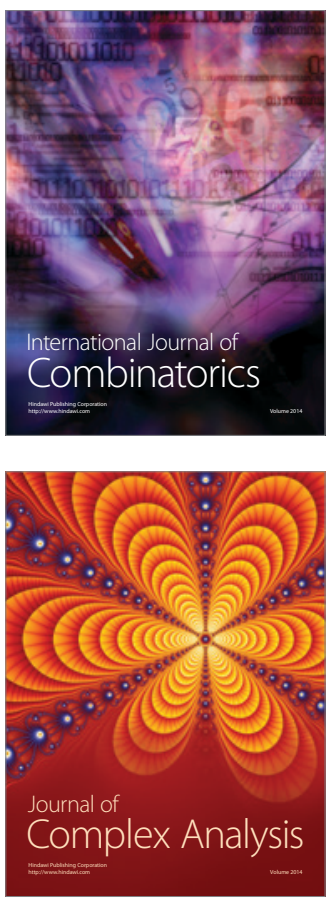

International Journal of

Mathematics and

Mathematical

Sciences
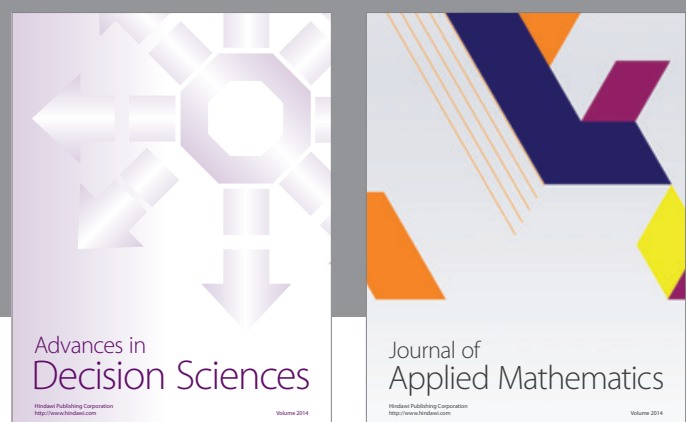

Journal of

Applied Mathematics
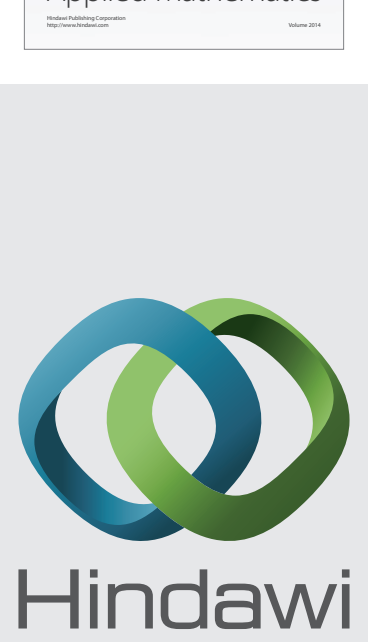

Submit your manuscripts at http://www.hindawi.com
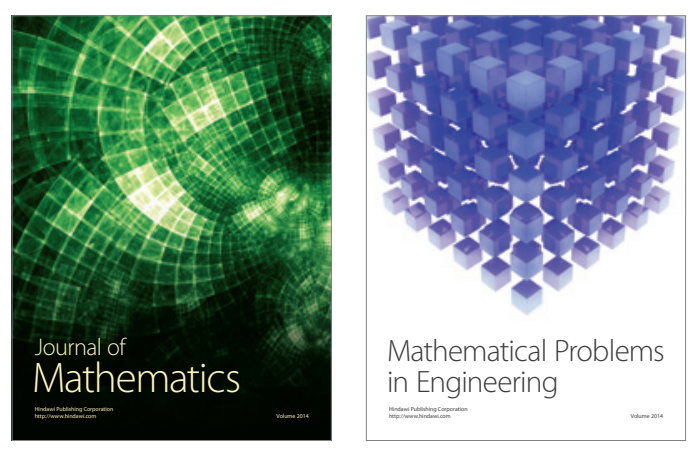

Mathematical Problems in Engineering
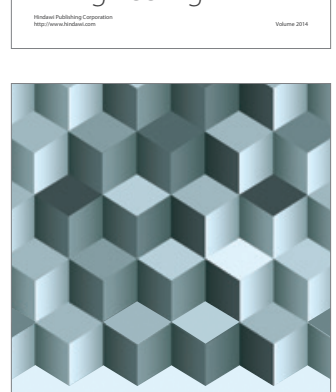

Journal of

Function Spaces
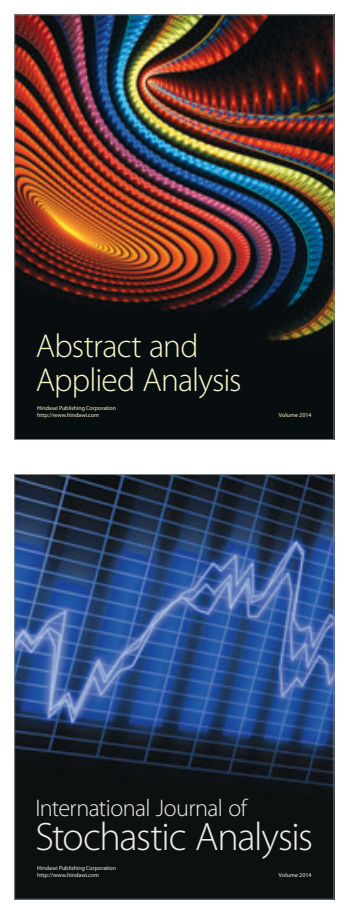

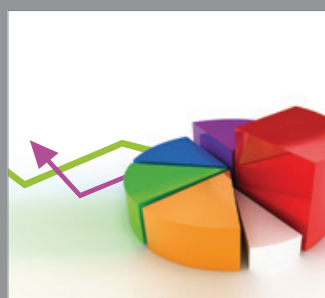

ournal of

Probability and Statistics

Promensencen
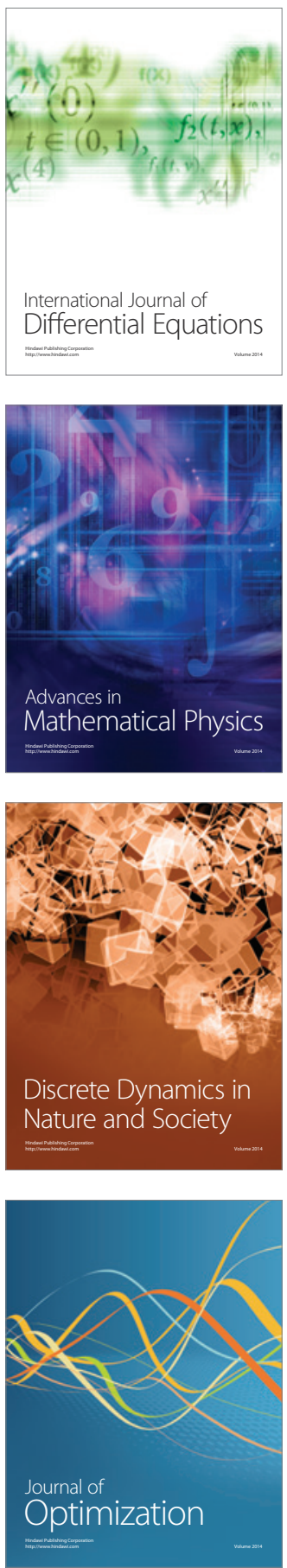